\title{
Aberrant expression and mechanism of miR-130b-3p/phosphatase and tensin homolog in nephroblastoma in children
}

\author{
YIFENG HU and JINGTIE YAN \\ Department of Pediatric Surgery, Zaozhuang Municipal Hospital, Zaozhuang, Shandong 277102, P.R. China
}

Received July 15, 2018; Accepted March 7, 2019

DOI: $10.3892 /$ etm.2019.7643

\begin{abstract}
Nephroblastoma is the most common renal tumor in children. Abnormal expression of microRNAs (miRs) has been reported to be involved in the progression of various types of cancers. However, the role and underlying mechanism of miR-130b-3p in nephroblastoma remains unknown. Therefore, the present study aimed to explore the role and possible mechanism of miR-130b-3p in nephroblastoma in children. The present study identified that miR-130b-3p was highly expressed in nephroblastoma tissues obtained from children with nephroblastoma. To better understand the functions and the molecular mechanisms of miR-130b-3p in nephroblastoma, TargetScan was used to identify the potential targets of miR-130b-3p. Phosphatase and tensin homolog (PTEN), was identified as a target gene of miR-130b-3p, and it was observed to be downregulated in nephroblastoma. Further analysis indicated that miR-130b-3p inhibitor could significantly reduce cell proliferation, induce apoptosis and suppress the Akt/nuclear factor- $\kappa \mathrm{B} /$ survivin signaling pathway in nephroblastoma cells. Notably, all these effects of miR-130b-3p on nephroblastoma cells were reversed by PTEN-small interfering RNA. In summary, the present study suggested that the miR-130b-3p/PTEN axis could serve a critical role in the progression and development of nephroblastoma. It also suggests that miR-130b-3p might be a valuable clinical biomarker and therapeutic target for nephroblastoma in children.
\end{abstract}

\section{Introduction}

Nephroblastoma is the most common pediatric kidney tumor with a prevalence of 1 in 10,000 children (1). Nephroblastoma originates from the embryo, develops in the renal parenchyma, undergoes a form distortion during growth, and invades the

Correspondence to: Dr Jingtie Yan, Department of Pediatric Surgery, Zaozhuang Municipal Hospital, 41 Longtouzhong Road, Zaozhuang, Shandong 277102, P.R. China

E-mail: jingtie201815@163.com

Key words: nephroblastoma, microRNA-130b-3p, phosphatase and tensin homolog, apoptosis surrounding kidney tissue. Currently, combination therapy has improved the prognosis of most patients, but there are still certain patients with poor survival due to recurrence and metastasis (2-5). Therefore, understanding the occurrence and development of nephroblastoma in children and seeking for new targets and methods for the treatment of nephroblastoma are essential.

MicroRNAs (miRNAs/miRs) are a group of highly conserved endogenous small non-coding RNAs (21-23 nucleotides in length) in eukaryotes (6). miRNAs do not encode proteins, and can inhibit target gene expression by binding to the 3 prime-untranslated region (3'-UTR) of the target gene (7-9). There is ample evidence indicating that miRNAs are involved in the regulation of multiple cellular events, including cell proliferation, differentiation, and apoptosis (10-12). In addition, abnormal expression of miRNAs has been shown to be involved in the development and progression of various types of tumors (9). miRNAs are promising biomarkers and therapeutic targets for cancer treatment.

miR-130b functions as an oncogenic factor or tumor suppressor miRNA, and shows dysregulation in various cancers including gliomas, gastric cancers, and endometrial cancers (13-15). Phosphatase and tensin homolog (PTEN) is one of the most common tumor suppressors in many human cancers and serves a vital role in the regulation of cell growth and apoptosis (16). In addition, it has been reported that PTEN is the target of various miRNAs. For example, miR-106b induces radio-resistance in colorectal cancer through the PTEN/Phophatidyl inositol 3'-OH kinase (PI3K)/Akt pathway (17). However, there is currently no report on the role of miR-130b-3p in nephroblastoma and the association of miR-130b-3p and PTEN in nephroblastoma.

Therefore, the present study aimed to investigate the role and underlying mechanism of miR-130b-3p/PTEN axis in nephroblastoma in children. The results of the present study may clarify the role and mechanism of miR-130b-3p in nephroblastoma and provide novel strategies and a theoretical basis for the treatment of nephroblastoma in children.

\section{Materials and methods}

Clinical samples. A total of 30 samples of nephroblastoma and normal adjacent tissues were obtained from children with nephroblastoma who had undergone surgery at the Department of Pediatric Surgery, Zaozhuang City Hospital 
between December 2015 and December 2017. All patients did not received chemotherapy or radiotherapy prior to surgery. The present study was approved by the Ethical Committee of the Zaozhuang City Hospital and informed consent was obtained from each child and their guardian.

Cell culture and cell transfection. Nephroblastoma cell line WiT49 was provided by The Hospital for Sick Children (Toronto, Canada) and was subsequently grown in Dulbecco's Modified Eagle's Medium/Nutrient Mixture F-12 (Invitrogen; Thermo Fisher Scientific, Inc.) containing 10\% fetal bovine serum (FBS; Invitrogen; Thermo Fisher Scientific, Inc.) and $1 \%$ penicillin streptomycin. These cells were cultured in a humidified chamber at $37^{\circ} \mathrm{C}$ with $5 \% \mathrm{CO}_{2}$. miR-130b-3p inhibitor (miR-130b-3p antagomir; sequence: 5'-UGCCAACCUUGCAAGCCGAAG-3') and inhibitor control (antagomir-negative control; sequence: 5'-CAGUACUUUUGUGUAGUACAA-3') were purchased from GenePharma Co. Ltd. WiT49 cells were transfected with either $100 \mathrm{nM}$ inhibitor control, $100 \mathrm{nM}$ miR-130b-3p inhibitor, $2 \mu \mathrm{l}$ control-siRNA (cat. no. Sc-36869; Santa Cruz Biotechnology, Inc., Dallas, TX, USA), $2 \mu$ PTEN-siRNA (cat. no. Sc-29459; Santa Cruz Biotechnology, Inc.), $100 \mathrm{nM}$ miR-130b-3p inhibitor $+2 \mu \mathrm{l}$ control-siRNA or $100 \mathrm{nM}$ miR-130b-3p inhibitor $+2 \mu \mathrm{l}$ PTEN-siRNA by using the Lipofectamine ${ }^{\circledR} 2000$ reagent (Invitrogen; Thermo Fisher Scientific, Inc.) according to the manufacturer's protocol. Cells without any treatment were considered as the control group. The cells were subjected to the following experiments $48 \mathrm{~h}$ after transfection.

Reverse transcription-quantitative polymerase chain reaction (RT-qPCR). The transfection efficiency was detected $48 \mathrm{~h}$ after cell transfection, using RT-qPCR and/or western blot assay. Total RNA from tissues and cells were extracted using the TRIzol Reagent (Takara Bio, Inc.) according to the manufacturer's protocol. The RNA concentration was detected by NanoDrop spectrophotometer (ND-2000; Nanodrop Technologies; Thermo Fisher Scientific, Inc.). The total RNA was stored at $-80^{\circ} \mathrm{C}$ until use. cDNAs were synthesized by using PrimeScript RT Reagent Kit (Takara Biotechnology Co., Ltd.) according to the manufacturer's protocol and analyzed by performing SYBR ${ }^{\circledR}$ Premix Ex Taq ${ }^{\mathrm{TM}}$ II (Takara Biotechnology Co., Ltd.) according to the manufacturer's protocol. The following primer sequences were used for qPCR: GAPDH forward, 5'-CGGGAAGCTTGTCATCAATGG-3' and reverse, 5'-GGCAGTGATGGCATGGACTG-3'; U6 forward, 5'-GCT TCGGCAGCACATATACTAAAAT-3' and reverse, 5'-CGC TTCACGAATTTGCGTGTCAT-3'; PTEN forward, 5'-GGA AAGGGACGAACTGGTGT-3' and reverse-5'-CAGGTA ACGGCTGAGGGAAC-3'; miR-130b-3p forward, 5'-CTG GTAGGGTACAGTACTGTGATA-3' and reverse, 5'-CTG GTGTCGTGGAGTCGGC-3'. The thermocycling conditions were as follows: $95^{\circ} \mathrm{C}$ for $5 \mathrm{~min}$, followed by 38 cycles of denaturation at $95^{\circ} \mathrm{C}$ for $15 \mathrm{sec}$ and annealing/elongation at $60^{\circ} \mathrm{C}$ for $30 \mathrm{sec}$. GAPDH (for mRNA) and U6 (for miRNA) were used as the internal controls. Relative expression levels were calculated by the $2^{-\Delta \Delta \mathrm{Cq}}$ method (18). All experiments were performed in triplicate.

Western blot assay. Radioimmunoprecipitation assay (RIPA) buffer (Beyotime Institute of Biotechnology) was used to extract proteins from cells or tissues according to the manufacturer's protocol. Bicinchoninic acid assay kit (Pierce Biotechnology; Thermo Fisher Scientific, Inc.) was used to quantify the protein samples in line with the manufacturer's protocol. The protein samples ( $25 \mu \mathrm{g} / \mathrm{lane})$ were separated on $10 \%$ SDS-PAGE gel and transferred to polyvinylidene difluoride membranes. The membranes were blocked with $5 \%$ non-fat milk for $1.5 \mathrm{~h}$ at room temperature, followed by incubation with primary antibody against PTEN (cat. no. 9188; 1:5,000; Cell Signaling Technology Inc.), Akt (cat. no. 4691; 1:5,000; Cell Signaling Technology Inc.), phospho-Akt-B (p-Akt; cat. no. 4060; 1:5,000; Cell Signaling Technology Inc.), nuclear factor (NF)-אB-p65 subunit (p65; cat. no. 8242; 1:5,000; Cell

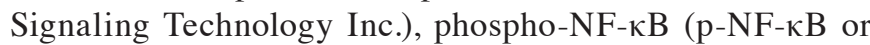
p-p65; cat. no. 3033; 1:5,000; Cell Signaling Technology Inc.), survivin (cat. no. 2808; 1:5,000; Cell Signaling Technology Inc.), or $\beta$-actin (cat. no. 4970; 1:5,000; Cell Signaling Technology Inc.) overnight at $4^{\circ} \mathrm{C}$. The membranes were then washed with Tris buffered saline with $0.1 \%$ Tween-20 (TBST) three times. Subsequently, the membranes were incubated with the anti-rabbit IgG, horse radish peroxidase-linked antibody (cat. no. 7074; 1:5,000; Cell Signaling Technology Inc.) for $2 \mathrm{~h}$ at room temperature. Finally, enhanced chemiluminescence reagent (Thermo Fisher Scientific, Inc.) was used to determine the immunoreactive bands. The band density was quantified with Gel-Pro Analyzer densitometry software (Version 6.3, Media Cybernetics, Inc.).

Cell proliferation assay. Cell proliferation was analyzed using the Cell Counting Kit-8 (CCK-8) assay (cat no. C0037; Beyotime Institute of Biotechnology). Logarithmic phase cells were seeded in a 96-well plate at a density of $1 \times 10^{4}$ cells per well and incubated in the $37^{\circ} \mathrm{C}, 5 \% \mathrm{CO}_{2}$ incubator for $12 \mathrm{~h}$. Subsequently, $10 \mu \mathrm{l} \mathrm{CCK}-8$ was added to each well, and the cells were incubated for a further $2 \mathrm{~h}$ at $37^{\circ} \mathrm{C}$ with $5 \% \mathrm{CO}_{2}$. The absorbance was measured at a wavelength of $450 \mathrm{~nm}$ using a microplate reader.

Flow cytometry assay. WiT49 cells were transfected with either $100 \mathrm{nM}$ inhibitor control, $100 \mathrm{nM}$ miR-130b-3p inhibitor, $100 \mathrm{nM}$ miR-130b-3p inhibitor $+2 \mu$ l control-siRNA or $100 \mathrm{nM}$ miR-130b-3p inhibitor $+2 \mu$ PTEN-siRNA for $48 \mathrm{~h}$. Then, WiT49 cells were collected by $0.25 \%$ Trypsin. Apoptotic cells were detected by using Annexin V-fluorescein isothiocyanate (FITC)/propidium iodide (PI) apoptosis detection kit (cat. no. 70-AP101-100; MultiSciences) according to the manufacturer's protocol. Briefly, cells $\left(10^{6}\right)$ were incubated with $5 \mu 1$ Annexin V-FITC and $5 \mu 1$ PI for $30 \mathrm{~min}$ in the absence of light at room temperature. Finally, flow cytometry (BD Biosciences) was used to detect cell apoptosis. The data were analyzed using WinMDI software (version 2.5; Purdue University Cytometry Laboratories; www.cyto.purdue. edu/flowcyt/software/Catalog.htm).

Dual-luciferase reporter analysis. TargetScan (www. targetscan.org) was used to predict the binding sites of miR-130b-3p and PTEN. To confirm the association between miR-130b-3p and PTEN, the dual-luciferase reporter vector pmiR-RB-REPORT ${ }^{\mathrm{TM}}$ (Guangzhou RiboBio Co., Ltd.) that contains a wild-type (WT) or mutant (MUT) 3'-UTR 
sequence of PTEN was constructed. Cells seeded in 24-well plates were co-transfected with miR-130b-3p mimics or mimic control and the MUT or WT 3'-UTR of PTEN using Lipofectamine ${ }^{\circledR} 2000$ reagent (Invitrogen; Thermo Fisher Scientific, Inc.). After transfection for $48 \mathrm{~h}$, cells were lysed with RIPA buffer. $48 \mathrm{~h}$ after transfection, the relative luciferase activity was detected using the Dual-Luciferase ${ }^{\circledR}$ Reporter Assay System (Promega Corporation, Madison, WI, USA) according to the manufacturer's protocol. Firefly luciferase activity was normalized to that of Renilla luciferase.

Statistical analyses. All experiments were performed in triplicates. All data were shown as the mean \pm standard deviation and SPSS v17.0 statistical software (SPSS, Inc.) was used for data analyses. Comparison between groups were performed by Student's t-tests and one-way analysis of variance followed by Tukey's test. $\mathrm{P}<0.05$ was considered to indicate a statistically significant difference.

\section{Results}

The expression of miR-130b-3p in nephroblastoma tissues. To explore the role of miR-130b-3p in nephroblastoma, RT-qPCR was performed to detect the relative expression of miR-130b-3p in nephroblastoma (tumor tissues) of children with nephroblastoma. The results revealed that compared with the adjacent tissues, the expression of miR-130b-3p was significantly upregulated in nephroblastoma tissues in children with nephroblastoma (Fig. 1).

PTEN is a direct target gene of $m i R-130 b-3 p$. TargetScan analysis was performed to identify functional targets of miR-130b-3p. Hundreds of target genes were identified to be the potential target genes of miR-130b-3p and as shown in Fig. 2A, PTEN was one such target. Based on the literature analysis, it was identified that PTEN is one of the most common tumor suppressor in many human cancers and serves a vital role in the regulation of cell growth and apoptosis $(16,19)$. Therefore, PTEN was selected for further investigations in the present study. Thus, to further examine whether miR-130b-3p directly targets PTEN, LucPTEN -3'UTR-WT and its 3'UTR-MUT plasmids were constructed. Luciferase reporter assay revealed that miR-130b-3p mimics clearly suppressed the luciferase activity of the WT-PTEN-3'UTR (Fig. 2B). Therefore, this provides evidence that PTEN is a direct target of miR-130b-3p.

The expression level of PTEN in nephroblastoma tissues. In order to examine the expression of PTEN in nephroblastoma tissues, RT-qPCR and western blot analysis were performed. The results demonstrated that compared with the adjacent tissues, PTEN was less expressed in nephroblastoma tissues, at the mRNA and protein expression level (three representative cases in each group are shown; Fig. 3A-C) in children. The mRNA (Fig. 3D) and protein expression (Fig. 3E and F) of PTEN in WT-CLS1 and WiT49 cells was determined using RT-qPCR and western blotting, respectively. The results indicated that the mRNA (Fig. 3D) and protein expression (Fig. 3E and F) of PTEN was higher in WiT49 cells than in

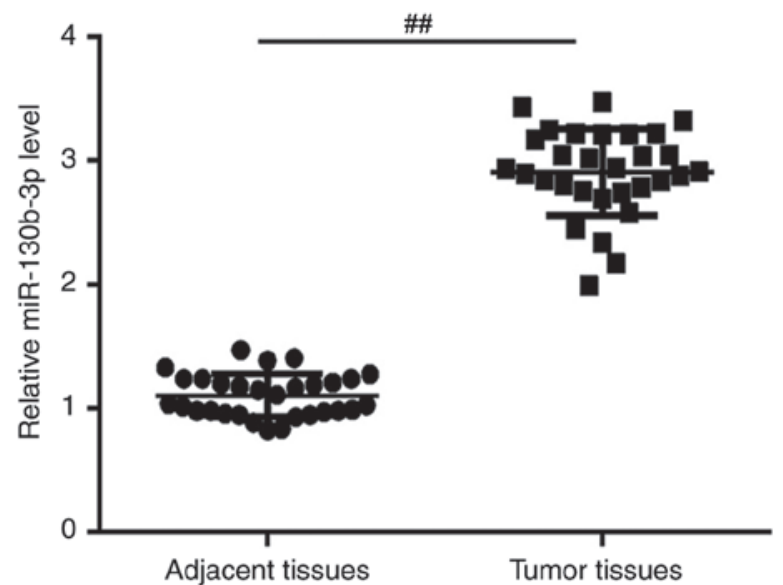

Figure 1. Expression levels of miR-130b-3p in nephroblastoma patients. The relative expression of miR-130b-3p in 30 cases of nephroblastoma and adjacent tissues of 30 children with nephroblastoma was detected using reverse transcription-quantitative polymerase chain reaction. Data are presented as the mean \pm standard deviation. ${ }^{\# \#} \mathrm{P}<0.01$ vs. adjacent tissues. miR, microRNA.

WT-CLS1 cells. WiT49 cells were then selected for further study.

Effect of miR-130b-3p on proliferation and apoptosis of nephroblastoma cells. WiT49 cells were transfected with inhibitor control or miR-130b-3p inhibitor for $48 \mathrm{~h}$ and RT-qPCR was performed to detect the efficiency of the inhibition. The results revealed that miR-130b-3p inhibitor significantly reduced miR-130b-3p expression in WiT49 cells (Fig. 4A). Furthermore, WiT49 cells were transfected with control-small interfering (si) RNA or PTEN-siRNA for $48 \mathrm{~h}$, and subsequently, RT-qPCR and western blotting were performed to detect the efficiency of siRNA. The results demonstrated that PTEN-siRNA reduced both mRNA and protein levels of PTEN in WiT49 cells (Fig. 4B-D). Finally, in order to test whether miR-130b-3p had an effect on PTEN expression in WiT49 cells, WiT49 cells were transfected with: Inhibitor control, miR-130b-3p inhibitor, miR-130b-3p inhibitor + control-siRNA, or miR-130b-3p inhibitor + PTEN-siRNA for $48 \mathrm{~h}$. RT-qPCR and western blotting were used to detect the expression of PTEN. It was revealed that miR-130b-3p inhibitor markedly increased PTEN expression levels at both mRNA and protein level, which was reversed by PTEN-siRNA (Fig. 4E-G).

In order to shed light on the function of miR-130b-3p in nephroblastoma, the effect of $\mathrm{miR}-130 \mathrm{~b}-3 \mathrm{p}$ on the cell proliferation of WiT49 cells was investigated. The results demonstrated that miR-130b-3p inhibitor significantly decreased WiT49 cell viability, which was reversed by PTEN-siRNA (Fig. 5A). To further determine whether miR-130b-3p could regulate WiT49 cell apoptosis, flow cytometry was performed to analyze apoptosis. The results revealed that miR-130b-3p inhibitor significantly induced WiT49 cell apoptosis, and this induction was reversed by PTEN-siRNA (Fig. 5B and C).

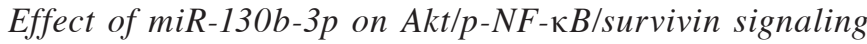
pathway in nephroblastoma cells. Finally, to explore the molecular mechanism of the effect of miR-130b-3p inhibitor on nephroblastoma cells, Akt/p-NF- $\mathrm{B} /$ survivin signaling 

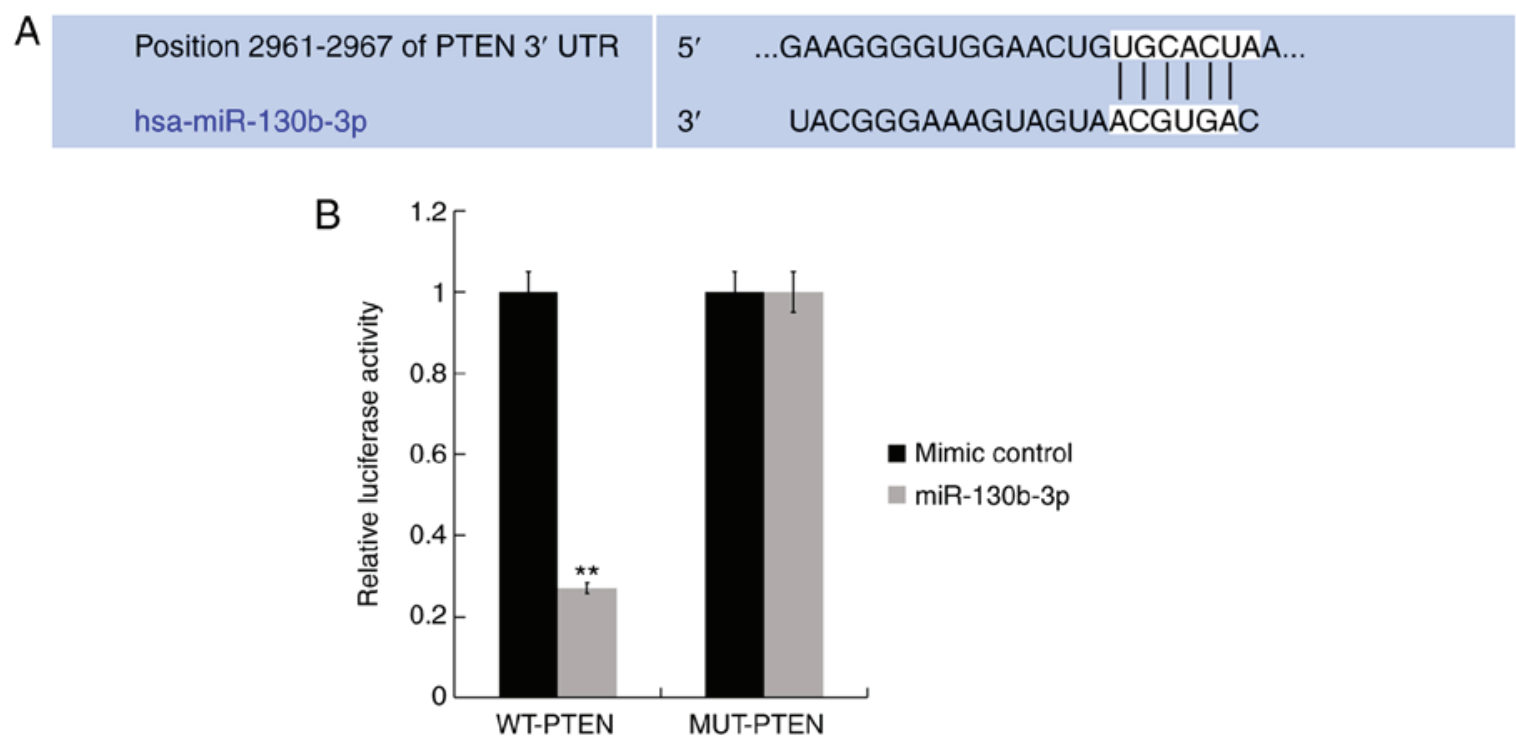

Figure 2. PTEN is a direct target of miR-130b-3p. (A) Interaction between miR-130b-3p and 3'-UTR of PTEN was predicted using a microRNA target site prediction software. (B) Luciferase activity of a reporter containing a WT-PTEN 3'-UTR or a MUT-PTEN 3'-UTR are presented. "* P $<0.01$ vs. mimic control. miR, microRNA; MUT, mutation in the miR-130b-3p binding site; PTEN, Phosphatase and tensin homolog; RT-qPCR, reverse transcription-quantitative polymerase chain reaction; UTR, untranslated region; WT, wild-type.

A
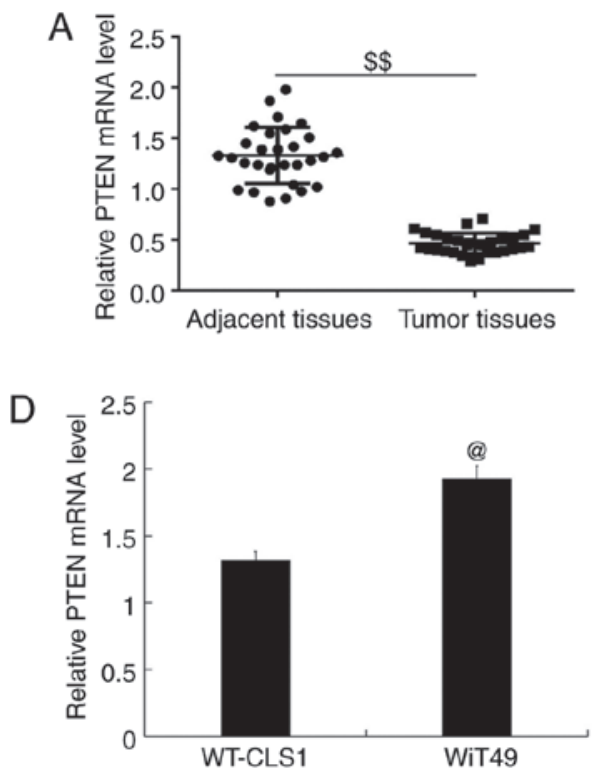
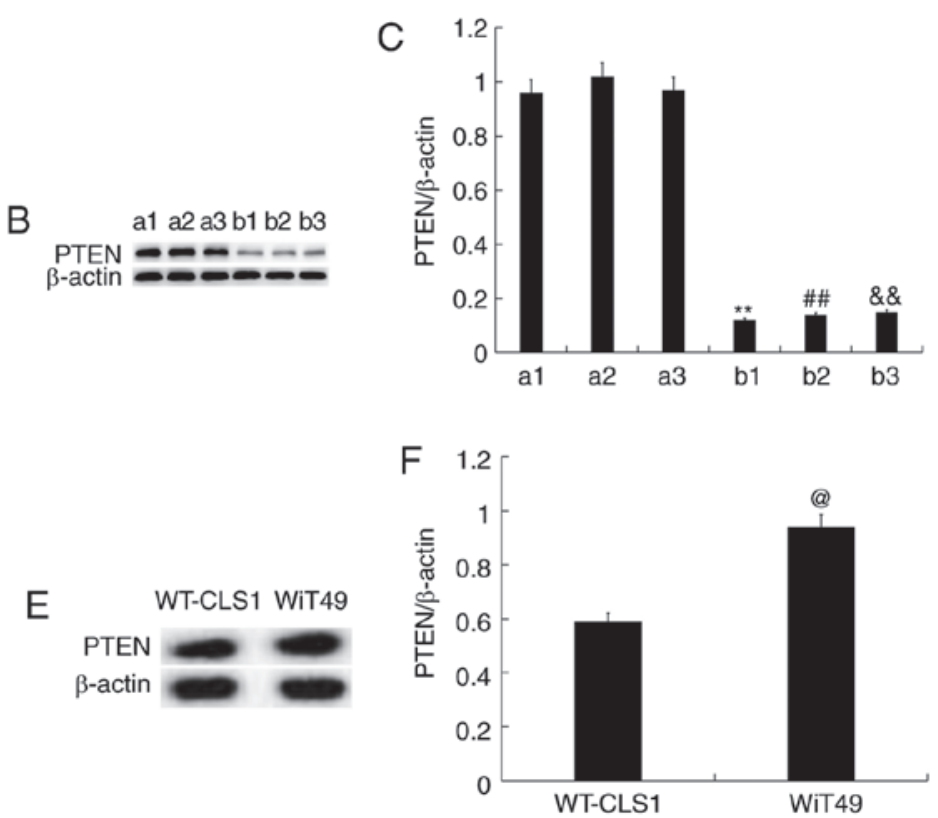

Figure 3. Expression levels of PTEN in nephroblastoma. (A) The relative mRNA expression of PTEN in nephroblastoma and adjacent tissues of children with nephroblastoma. (B) and (C) The protein expression levels of PTEN in nephroblastoma and adjacent tissues of children with nephroblastoma (three representative cases are shown for protein. a1, a2, a3 indicate 3 cases of tumor tissues of three children with nephroblastoma; b1, b2, b3 indicate the paired adjacent tissues). (D-E) The mRNA and protein level of PTEN in WT-CLS1 and WiT49 cells was detected using RT-qPCR and western blot assay .(F) The ratio of PTEN/ $\beta$-actin was calculated and presented. ${ }^{\circledR} \mathrm{P}<0.05$ vs. WT-CLS1. ${ }^{\$ \$} \mathrm{P}<0.01$ vs. adjacent tissues, ${ }^{* *} \mathrm{P}<0.01$ vs. a1, ${ }^{\# \#} \mathrm{P}<0.01$ vs. a2, ${ }^{\star} \mathrm{P}<0.01 \mathrm{vs}$. a3. PTEN, Phosphatase and tensin homolog; WT, wild-type.

pathway was analyzed. As shown in Fig. 6A, compared with the control group, the protein levels of p-Akt, p-NF- $\mathrm{B}$ (p-p65) were markedly decreased by miR-130b-3p inhibitor, and these decreases were eliminated by PTEN silencing. The ratio of p-Akt/total-Akt and p-p65/p65 significantly decreased following treatment with the miR-130b-3p inhibitor, and these decreases were ameliorated following PTEN silencing (Fig. 6B and C). Furthermore, survivin protein levels were significantly decreased by miR-130b-3p inhibitor treatment.
However, these decreases were reversed following PTEN silencing (Fig. 6D and E).

\section{Discussion}

miRNAs have emerged as promising biomarkers for tumors (20-22). It has been reported that aberrant expression of miRNAs participated in physiological and pathological processes of a variety of human cancers, 

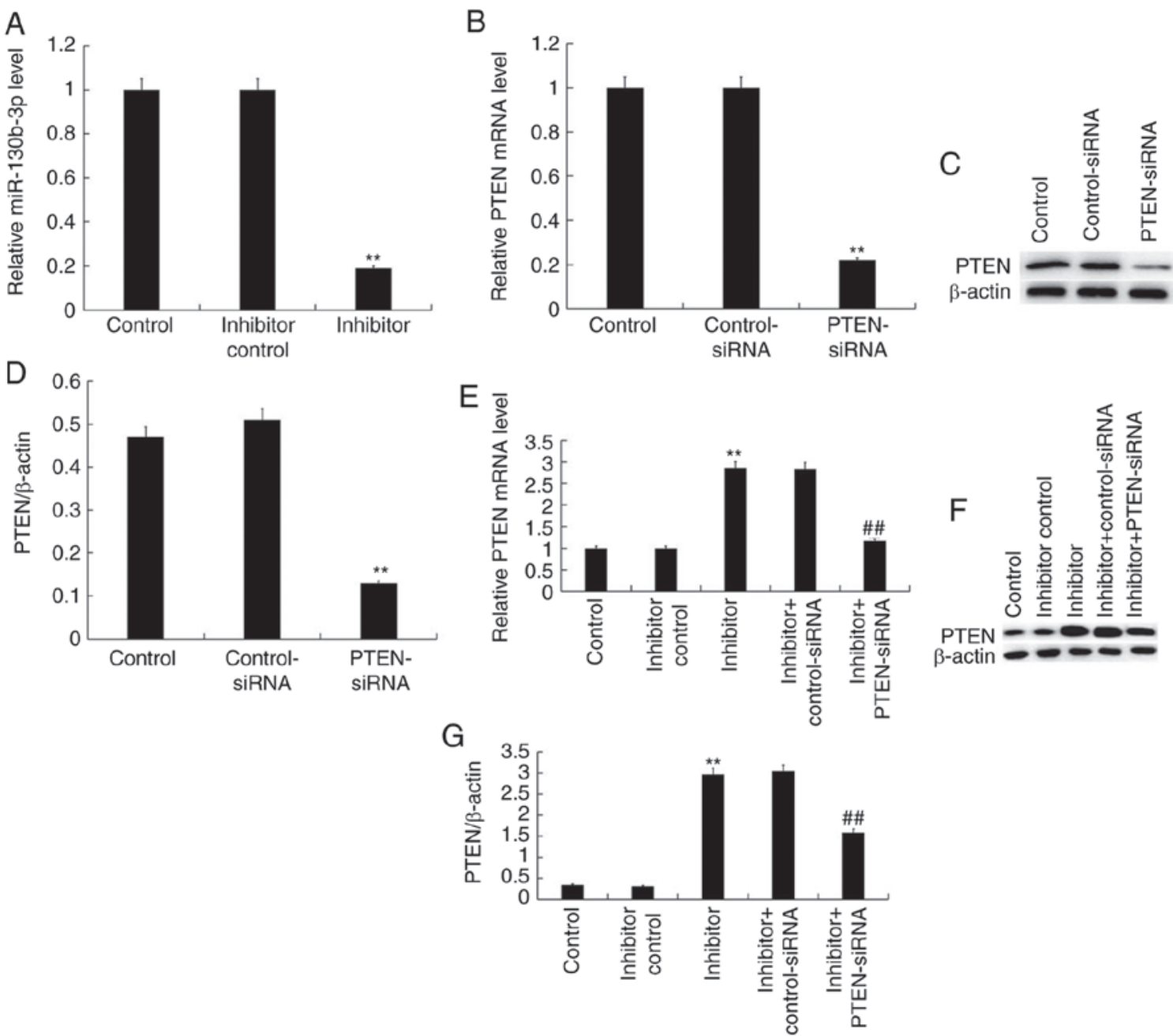

Figure 4. miR-130b-3p regulates the expression of PTEN in WiT49 cells. (A) The mRNA expression levels of miR-130b-3p after WiT49 cells transfected with control, inhibitor control and miR-130b-3p inhibitor for 48 h. (B) mRNA expression levels of PTEN following transfection with control, control-siRNA or PTEN-siRNA. (C) PTEN protein expression levels and (D) normalized to $\beta$-actin in different groups. (E) PTEN mRNA expression levels after transfection with control, inhibitor control, miR-130b-3p inhibitor, inhibitor + control-siRNA or inhibitor + PTEN-siRNA (F) PTEN expression levels and (G) normalized to $\beta$-actin in different groups. Expression levels were measured by reverse transcription-quantitative polymerase chain reaction/western blotting. Data presented as the mean \pm standard deviation. ${ }^{* *} \mathrm{P}<0.01$ vs. control, ${ }^{* \#} \mathrm{P}<0.01$ vs. inhibitor. miR, microRNA; PTEN, Phosphatase and tensin homolog; RT-qPCR, reverse transcription-quantitative polymerase chain reaction; siRNA, small interfering RNA.
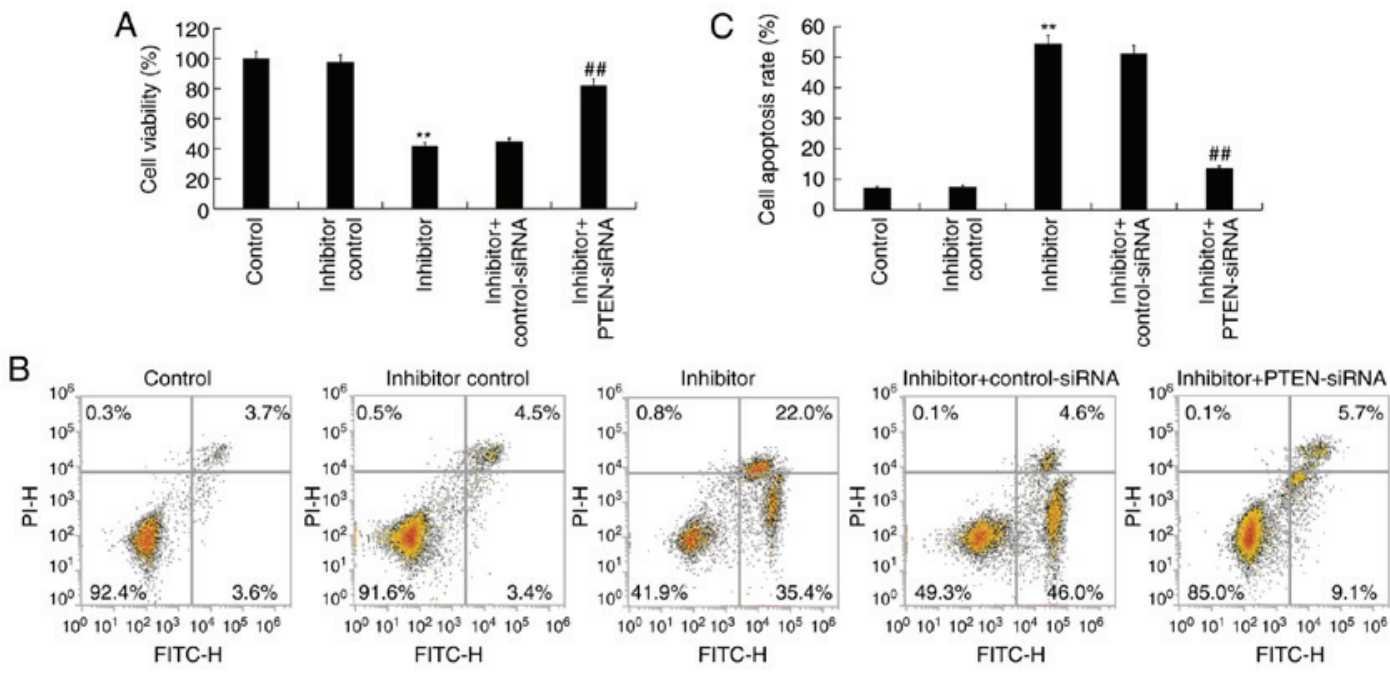

Figure 5. Effect of miR-130b-3p on nephroblastoma cell viability and apoptosis. (A) Cell viability of WiT49 cells detected using Cell counting kit-8 assay. (B) Cell apoptosis was analyzed using flow cytometry and (C) quantification of the total apoptosis rate. Data are presented as the mean \pm standard deviation. ${ }^{*} \mathrm{P}<0.01$ vs. control. ${ }^{\# /} \mathrm{P}<0.01$ vs. inhibitor. FITC, fluorescein isothiocyanate; PI, propidium iodide; miR, micro RNA; siRNA, small interfering RNA; PTEN, Phosphatase and tensin homolog. 

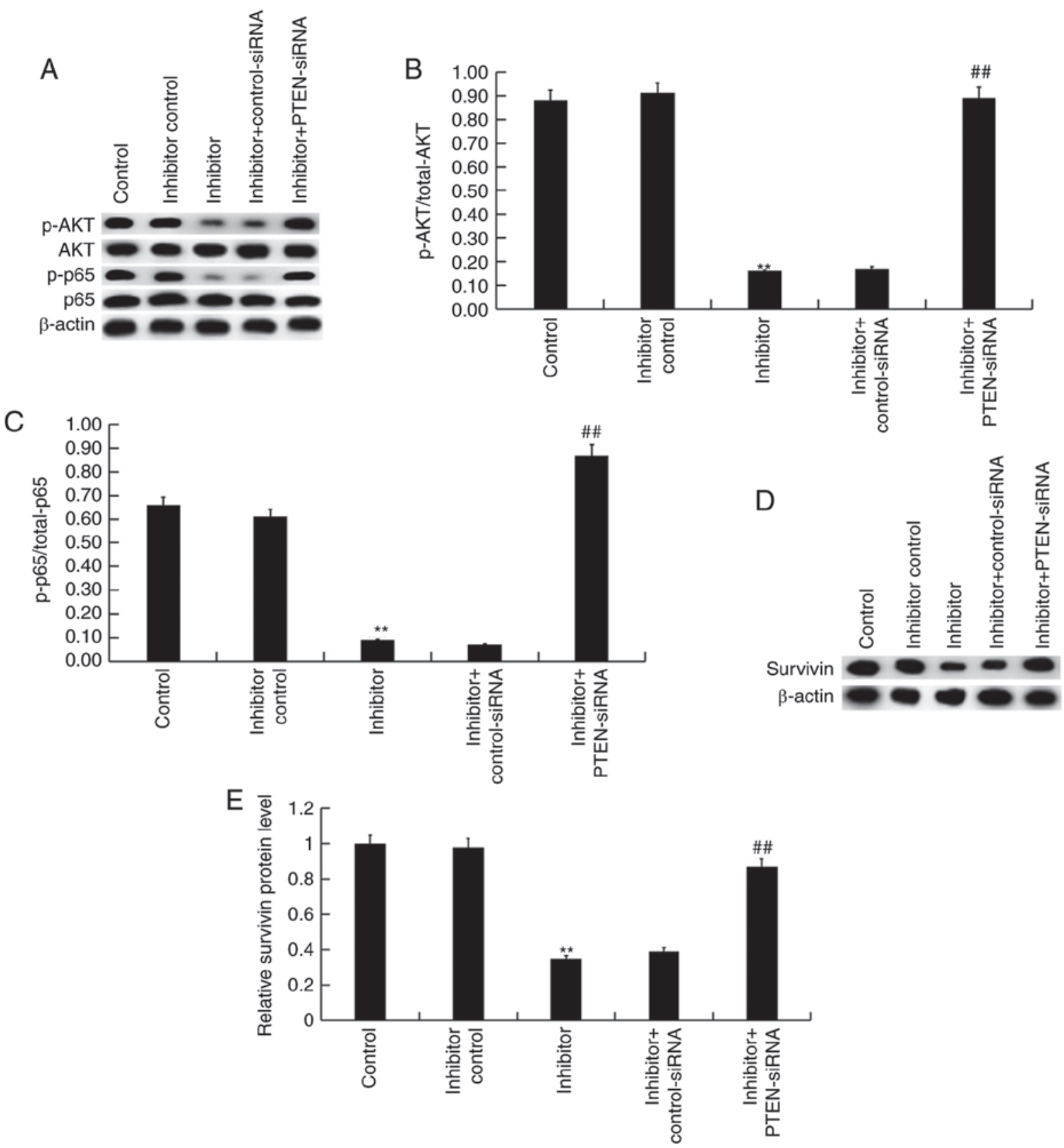

Figure 6. Effect of miR-130b-3p on Akt/p-NF- $\mathrm{kB} /$ survivin signaling pathway in nephroblastoma cells after $48 \mathrm{~h}$ transfection. (A) The protein levels of Akt, p-Akt, p65, p-p65 were measured by western blot assay. (B and C) Expression levels of p-Akt/total-Akt and p-p65/total-p65 were calculated. (D) Survivin expression level was measured using western blotting and (E) quantification of its expression level. Data are presented as the mean \pm standard deviation. ${ }^{* *} \mathrm{P}<0.01$ vs. control. ${ }^{\# / P}<0.01$ vs. inhibitor. miR, microRNA; PTEN, Phosphatase and tensin homolog; siRNA, small interfering RNA.

including proliferation (23), invasion (24), apoptosis (25) and chemotherapy resistance (26). In addition, miRNA dysregulation is causally involved in the initiation and progression of cancer (27-29). Furthermore, miRNAs can act as either oncogenes or tumor suppressors through interaction with specific targets $(30,31)$.

A series of microarray chips have been used to detect the miRNA expression of nephroblastoma and these studies have indicated the abnormal expression levels of various miRNAs in nephroblastoma, including the upregulated genes, miR-378 and miR-18b, and the downregulated genes, miR-193a-5p and miR-199a-5p (5,32). Zhu et al (33) demonstrated that miR-92a-3p was downregulated in nephroblastoma, and it inhibited nephroblastoma cell proliferation, colony formation, migration and invasion of nephroblastoma by targeting Notch1. The present study revealed that miR-130b-3p was upregulated in nephroblastoma tissues. A large number of studies have indicated that miR-130b-3p was associated with various cancers, including breast cancer (34), lung cancer (35), bladder carcinoma (36), and human epithelial ovarian cancer (37).

A previous study demonstrated that miR-130b-3p targets PTEN to mediate chemoresistance, proliferation and apoptosis via Wnt/ $\beta$-catenin pathway in lung cancer cells (35). It has been reported that miR-130b-3p targets PTEN to promote proliferation and decrease apoptosis by the PI3K/Akt signaling pathway in breast cancer cells (34). The results of the present study confirmed that PTEN was a direct target gene of miR-130b-3p in nephroblastoma cells, which were 
consistent with a previous study (38). The expression of PTEN was markedly decreased in nephroblastoma tissues and cells. Furthermore, the present study demonstrated that the inhibitor of miR-130b-3p, inhibited proliferation, promoted apoptosis and repressed the Akt/p-NF- $\mathrm{B} /$ survivin signaling pathway in nephroblastoma cells. It is worth mentioning that all the effects of miR-130b-3p inhibitor on nephroblastoma cells were eliminated by PTEN silencing.

In conclusion, the present study indicated that miR-130b-3p was upregulated in nephroblastoma, and its downregulation could inhibit proliferation and induce apoptosis through repressing $\mathrm{Akt} / \mathrm{p}-\mathrm{NF}-\kappa \mathrm{B} /$ survivin signaling pathway by targeting PTEN in nephroblastoma cells. These findings may provide important theoretical basis and therapeutic strategies for nephroblastoma. However, this is a preliminary study of the role of miR-130b-3p in nephroblastoma and the effect of miR-130b-3p overexpression on nephroblastoma cells is still unclear. Therefore, in order to fully understand the role of miR-130b-3p in nephroblastoma, and to ascertain whether miR-130b-3p has a similar effect on nephroblastoma in vivo requires further study. Furthermore, the association of miR-130b-3p expression with the clinical characteristics and prognosis of children with nephroblastoma is still unclear. Thus, further in vivo and clinical studies are required to prove the role of miR-130b-3p in nephroblastoma.

\section{Acknowledgements}

Not applicable.

\section{Funding}

No funding was received.

\section{Availability of data and materials}

All data sets used and/or generated during the current study are available from the corresponding author on reasonable request.

\section{Authors' contributions}

YH designed the current study. YH and JY analyzed the data and prepared the manuscript. All authors read and approved the final manuscript.

\section{Ethics approval and consent to participate}

The present study was approved by the Ethical Committee of the Zaozhuang Municipal Hospital (Zaozhuang, China) and informed consent was obtained from each child and their guardian prior to enrollment.

\section{Patient consent for publication}

Not applicable.

\section{Competing interests}

The authors declare that they have no competing interests.

\section{References}

1. Charlton J, Pavasovic V and Pritchard-Jones K: Biomarkers to detect Wilms tumors in pediatric patients: Where are we now? Future Oncol 11: 2221-2234, 2015.

2. Cone EB, Dalton SS, Van Noord M, Tracy ET, Rice HE and Routh JC: Biomarkers for Wilms tumor: A systematic review. J Urol 196: 1530-1535, 2016.

3. Qi C, Hu Y, Yang F, An H, Zhang J, Jin H and Guo F: Preliminary observations regarding the expression of collagen triple helix repeat-containing 1 is an independent prognostic factor for Wilms' tumor. J Pediatr Surg 51: 1501-1506, 2016.

4. Brok J, Pritchard-Jones K, Geller JI and Spreafico F: Review of phase I and II trials for Wilms' tumour-Can we optimise the search for novel agents? Eur J Cancer 79: 205-213, 2017.

5. Yu X, Li Z, Chan MT and Wu WK: The roles of microRNAs in Wilms' tumors. Tumour Biol 37: 1445-1450, 2016.

6. Li X, Liu F, Lin B, Luo H, Liu M, Wu J, Li C, Li R, Zhang X, Zhou K and Ren D: miR-150 inhibits proliferation and tumorigenicity via retarding G1/S phase transition in nasopharyngeal carcinoma. Int J Oncol 50: 1097-1108, 2017.

7. Ro S, Park C, Young D, Sanders KM and Yan W: Tissue-dependent paired expression of miRNAs. Nucleic Acids Res 35: 5944-5953, 2007.

8. Mallory AC and Vaucheret H: MicroRNAs: Something important between the genes. Curr Opin Plant Biol 7: 120-125, 2004.

9. Garzon R, Calin GA and Croce CM: MicroRNAs in cancer. Annu Rev Med 60: 167-179, 2009.

10. Ebert MS and Sharp PA: Roles for microRNAs in conferring robustness to biological processes. Cell 149: 515-524, 2012.

11. Rogers $\mathrm{K}$ and Chen X: Biogenesis, turnover, and mode of action of plant microRNAs. Plant Cell 25: 2383-2399, 2013.

12. Hayes J, Peruzzi PP and Lawler S: MicroRNAs in cancer: Biomarkers, functions and therapy. Trends Mol Med 20: 460-469, 2014.

13. Xiao ZQ, Yin TK, Li YX, Zhang JH and Gu JJ: miR-130b regulates the proliferation, invasion and apoptosis of glioma cells via targeting of CYLD. Oncol Rep 38: 167-174, 2017.

14. Sun B, Li L, Ma W, Wang S and Huang C: MiR-130b inhibits proliferation and induces apoptosis of gastric cancer cells via CYLD. Tumour Biol 37: 7981-7987, 2016.

15. Li BL, Lu C, Lu W, Yang TT, Qu J, Hong X and Wan XP: miR-130b is an EMT-related microRNA that targets DICER1 for aggression in endometrial cancer. Med Oncol 30: 484, 2013.

16. Di Cristofano A and Pandolfi PP: The multiple roles of PTEN in tumor suppression. Cell 100: 387-390, 2000.

17. Zheng L, Zhang Y, Liu Y, Zhou M, Lu Y, Yuan L, Zhang C, Hong $\mathrm{M}$, Wang S and Li X: MiR-106b induces cell radioresistance via the PTEN/PI3K/Akt pathways and p21 in colorectal cancer. J Transl Med 13: 252, 2015.

18. Livak KJ and Schmittgen TD: Analysis of relative gene expression data using real-time quantitative PCR and the 2(-Delta Delta C(T)) method. Methods 25: 402-408, 2001.

19. Zhou X, Yang X, Sun X, Xu X, Li X, Guo Y, Wang J, Li X, Yao L, Wang $\mathrm{H}$ and Shen L: Effect of PTEN loss on metabolic reprogramming in prostate cancer cells. Oncol Lett 17: 2856-2866, 2019.

20. Shin VY and Chu KM: MiRNA as potential biomarkers and therapeutic targets for gastric cancer. World J Gastroenterol 20: 10432-10439, 2014.

21. Rocci A, Hofmeister CC and Pichiorri F: The potential of miRNAs as biomarkers for multiple myeloma. Expert Rev Mol Diagn 14: 947-959, 2014.

22. Galardi A, Colletti M, Businaro P, Quintarelli C, Locatelli F and Di Giannatale A: MicroRNAs in neuroblastoma: Biomarkers with therapeutic potential. Curr Med Chem 25: 584-600, 2018.

23. Yuan Y, Anbalagan D, Lee LH, Samy RP, Shanmugam MK, Kumar AP, Sethi G, Lobie PE and Lim LH: ANXA1 inhibits miRNA-196a in a negative feedback loop through NF-kB and c-Myc to reduce breast cancer proliferation. Oncotarget 7: 27007-27020, 2016.

24. Yang L, Li Q, Wang Q, Jiang Z and Zhang L: Silencing of miRNA-218 promotes migration and invasion of breast cancer via Slit2-Robo1 pathway. Biomed Pharmacother 66: 535-540, 2012.

25. Li P, Xie XB, Chen Q, Pang GL, Luo W, Tu JC, Zheng F, Liu SM, Han L, Zhang JK, et al: MiRNA-15a mediates cell cycle arrest and potentiates apoptosis in breast cancer cells by targeting synuclein- $\gamma$. Asian Pac J Cancer Prev 15: 6949-6954, 2014. 
26. Kastl L, Brown I and Schofield AC: miRNA-34a is associated with docetaxel resistance in human breast cancer cells. Breast Cancer Res Treat 131: 445-454, 2012.

27. Calin GA and Croce CM: MicroRNA signatures in human cancers. Nat Rev Cancer 6: 857-866, 2006.

28. Esquela-Kerscher A and Slack FJ: Oncomirs-microRNAs with a role in cancer. Nat Rev Cancer 6: 259-269, 2006.

29. Kent OA and Mendell JT: A small piece in the cancer puzzle: MicroRNAs as tumor suppressors and oncogenes. Oncogene 25: 6188-6196, 2006.

30. Bartel DP: MicroRNAs: Genomics, biogenesis, mechanism, and function. Cell 116: 281-297, 2004.

31. Lagos-Quintana M, Rauhut R, Lendeckel W and Tuschl T: Identification of novel genes coding for small expressed RNAs. Science 294: 853-858, 2001.

32. Watson JA, Bryan K, Williams R, Popov S, Vujanic G, Coulomb A, Boccon-Gibod L, Graf N, Pritchard-Jones K and O'Sullivan M: miRNA profiles as a predictor of chemoresponsiveness in Wilms' tumor blastema. PLoS One 8: e53417, 2013.

33. Zhu S, Zhang L, Zhao Z, Fu W, Fu K, Liu G and Jia W: microRNA-92a-3p inhibits the cell proliferation, migration and invasion of Wilms tumor by targeting NOTCH1. Oncol Rep 40: $571-578,2018$

34. Miao Y, Zheng W, Li N, Su Z, Zhao L, Zhou H and Jia L: MicroRNA-130b targets PTEN to mediate drug resistance and proliferation of breast cancer cells via the PI3K/Akt signaling pathway. Sci Rep 7: 41942, 2017.
35. Zhang Q, Zhang B, Sun L, Yan Q, Zhang Y, Zhang Z, Su Y and Wang C: MicroRNA-130b targets PTEN to induce resistance to cisplatin in lung cancer cells by activating $\mathrm{Wnt} / \beta$-catenin pathway. Cell Biochem Funct 36: 194-202, 2018.

36. Lv M, Zhong Z, Chi H, Huang $M$, Jiang $R$ and Chen J: Genome-wide screen of miRNAs and targeting mRNAs reveals the negatively regulatory effect of miR-130b-3p on PTEN by $\mathrm{PI} 3 \mathrm{~K}$ and integrin $\beta 1$ signaling pathways in bladder carcinoma. Int J Mol Sci 18: 78, 2017.

37. Zhou D, Zhang L, Sun W, Guan W, Lin Q, Ren W, Zhang J and $\mathrm{Xu}$ G: Cytidine monophosphate kinase is inhibited by the TGF- $\beta$ signalling pathway through the upregulation of miR-130b-3p in human epithelial ovarian cancer. Cell Signal 35: 197-207, 2017.

38. Yu T, Cao R, Li S, Fu M, Ren L, Chen W, Zhu H, Zhan Q and Shi R: MiR-130b plays an oncogenic role by repressing PTEN expression in esophageal squamous cell carcinoma cells. BMC Cancer 15: 29, 2015. International (CC BY-NC-ND 4.0) License. 